Discrete Comput Geom 36:503-509 (2006)

DOI: $10.1007 / \mathrm{s} 00454-006-1262-\mathrm{y}$

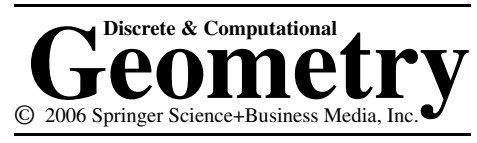

\title{
On Distinct Distances from a Vertex of a Convex Polygon
}

\author{
Adrian Dumitrescu \\ Computer Science, University of Wisconsin-Milwaukee, \\ 3200 N. Cramer Street, Milwaukee, WI 53211, USA \\ ad@cs.uwm.edu \\ Dedicated to János Pach on the occasion of his 50th birthday
}

\begin{abstract}
Given a set $P$ of $n$ points in convex position in the plane, we prove that there exists a point $p \in P$ such that the number of distinct distances from $p$ is at least $\lceil(13 n-6) / 36\rceil$. The best previous bound, $\lceil n / 3\rceil$, from 1952 , is due to Moser.
\end{abstract}

\section{Introduction}

A set of points in the plane is in general position if no three points are collinear. A finite set of points is in convex position if the points are the vertices of a convex polygon.

The following famous problem was raised by Erdős [3] in 1946. What is the minimum number of distinct distances determined by $n$ points in the plane? Denoting this number by $g(n)$, he conjectured that $g(n)=\Omega(n / \sqrt{\log n})$, and showed that this bound is attained by the $\sqrt{n} \times \sqrt{n}$ integer grid. The best known lower bound, $\approx \Omega\left(n^{0.8641}\right)$, is the last in a sequence of recent results due to Solymosi and C. Tóth [13], Tardos [14], and Katz and Tardos [9]. See also the related work of Pach and Tardos [12].

If the $n$ points are in convex position, then the minimum number of distinct distances determined is much larger. The following classical result of Moser [10] is well known:

Theorem 1 (Moser). Let $P$ be a set of $n$ points in convex position in the plane. Then there exists a point $p \in P$ such that the number of distinct distances from $p$ is at least $\lceil n / 3\rceil$.

Following in part the presentation in Pach and Agarwal's book [11, page 206], let $g^{\text {conv }}(n)$ denote the minimum number of distinct distances determined by a set of $n$ points 
in convex position. Altman [1], [2] proved that

$$
g^{\text {conv }}(n)=\left\lfloor\frac{n}{2}\right\rfloor .
$$

The bound is tight, and attained for a regular $n$-gon.

Erdós conjectured that the following stronger statement is also true. Every convex $n$-gon has a vertex such that the number of distinct distances from this vertex is at least $\lfloor n / 2\rfloor$. If true, this would be again best possible, as a regular $n$-gon shows. We make a step in this direction by improving the bound in Theorem 1, a result which is by now more than 50 years old. Somewhat surprisingly, most of the ingredients present in our proof were known for many years.

Theorem 2. Let $P$ be a set of $n$ points in convex position in the plane. Then there exists a point $p \in P$ such that the number of distinct distances from $p$ is at least $\lceil(13 n-6) / 36\rceil$.

As mentioned in [11], it seems likely that the above result of Altman can be generalized to any set of points in general position. That is, if $g^{\text {gen }}(n)$ denotes the minimum number of distinct distances determined by a set of $n$ points in general position in the plane, it can be conjectured that

$$
g^{\text {gen }}(n)=g^{\text {conv }}(n)=\left\lfloor\frac{n}{2}\right\rfloor .
$$

The best result in this direction is due to Szemerédi [4] (see also page 207 of [11]):

Theorem 3 (Szemerédi). Let $P$ be a set of $n$ points in general position in the plane. Then there exists a point $p \in P$ such that the number of distinct distances from $p$ is at least $\lceil(n-1) / 3\rceil$.

Note that the above bound for points in general position is essentially the same as that for points in convex position (Theorem 1), from which it can differ by at most one. Our Theorem 2 introduces some distinction between the results for points in general position and the presumably easier case of points in convex position.

A result related to Theorem 1 is the following. Let $h(n)$ be the largest integer $h$ such that every convex polygon with $n$ vertices has a vertex $p$ so that the next $h$ vertices clockwise from $p$, or the next $h$ vertices counterclockwise from $p$, are successively farther from $p$. Erdôs and Fishburn [5] have proved that $h(n)=\lfloor n / 3\rfloor+1$ for $n \geq 4$. Their proof of the lower bound slightly extends Moser's approach which was showing that $h(n) \geq\lceil n / 3\rceil$ (and thereby improves Moser's lower bound by one in the case when $n$ is a multiple of three).

\section{Proof of Theorem 2}

Let $C$ be a circular disk, and let $p q$ be a chord of $C$. The chord $p q$ divides the disk into two parts, the smaller of which is called a cap; if $p q$ is a diameter of $C$, then both parts are considered caps. Lemma 1 below is used by Moser in proving his $\lceil n / 3\rceil$ lower bound (see page 206 of [11]). 
Lemma 1 [10]. Let $P$ be a set of $m$ points in convex position inside a closed cap of a disk $C$ determined by a chord $p q$, such that $p, q \in P$. Then all the $m-1$ distances between $p$ and the other points of $P$ are distinct.

The first part in our proof is the same as in Moser's proof (see pages 206-207 of [11]). Let $C$ be the smallest disc containing all points in $P$. If only two points $p, q \in P$ lie on the boundary of $C$, then $p q$ must be a diameter, and at least one of the two closed caps determined by $p q$ contains at least $\lceil n / 2\rceil+1$ points of $P$. By Lemma 1 , there are at least $\lceil n / 2\rceil$ distinct distances from $p$, and Theorem 2 follows in this case.

If $C$ has more than two points on its boundary, then one can choose three of them, $p, q, r \in P$, so that no angle of $\Delta p q r$ is obtuse (for otherwise $C$ would not be minimum; the detailed argument appears in [10]). Since $P$ is in convex position, no element of $P$ is in the interior of $\Delta p q r$. Thus, all of $P$ is contained in the three circular caps $C_{1}, C_{2}$, and $C_{3}$ determined by the chords $p q, q r$, and $r p$, respectively. Denote by $m_{i}$ the number of points in cap $C_{i}, i=1,2,3$. Then

$$
m_{1}+m_{2}+m_{3}=n+3 .
$$

We now adapt Szemerédi's proof to the situation when the points are in convex position and are included in the three caps as outlined above. The original proof (conforming with the presentation on pages 207-208 of [11]) goes as follows.

Assume that for every point $p \in P$, the number of distinct distances from $p$ is at most $k$. This means that every point $q \neq p$ lies on one of at most $k$ concentric circles $C_{1}(p), \ldots, C_{k}(p)$ centered at $p$. Let $I$ be the number of isosceles triangles determined by $P$, where an equilateral triangle is counted with multiplicity three. Then,

$$
I=\sum_{p \in P} \sum_{i=1}^{k}\left(\begin{array}{c}
\left|C_{i}(p) \cap P\right| \\
2
\end{array}\right)
$$

which attains its minimum if the points of $P \backslash\{p\}$ are distributed among the circles $C_{i}(p)$ as uniformly as possible. In particular,

$$
I \geq n k \frac{n-1}{k}\left(\frac{n-1}{k}-1\right) / 2 .
$$

On the other hand, every segment $q r$ can be the base of at most two isosceles triangles determined by $P$, for otherwise the perpendicular bisector of $q r$ would pass through at least three points of $P$. Thus,

$$
I \leq 2\left(\begin{array}{l}
n \\
2
\end{array}\right)
$$

Putting these two inequalities together, one gets $k \geq\lceil(n-1) / 3\rceil$.

We now return to the proof of Theorem 2, and first improve the upper bound on $I$. Two key facts in our proof are the following easy generalization of Lemma 1 and its corollary: 


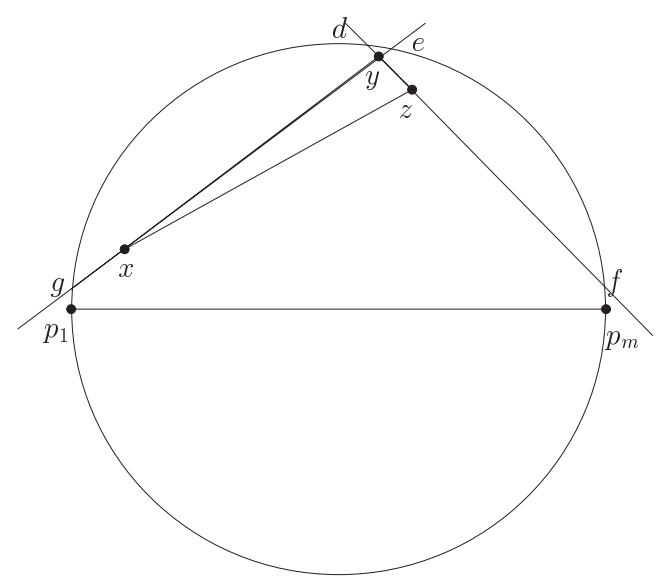

Fig. 1. Points in a circular cap; proof of Lemma 2.

Lemma 2. Let $P=\left\{p_{1} \cdots p_{m}\right\}$ be a set of $m$ points in convex position inside a closed cap of a disk $C$ determined by chord $p_{1} p_{m}$, where the points are labeled in clockwise order. Then for any $i \in\{1, \ldots, m\}$, (a) all the $m-i$ distances between $p_{i}$ and the points $p_{j}(j>i)$ of $P$ (which follow it in clockwise order) are distinct; $(\mathrm{b})$ all the $i-1$ distances between $p_{i}$ and the points $p_{j}(j<i)$ of $P$ (which precede it in clockwise order) are distinct.

Proof. For simplicity assume that $p_{1} p_{m}$ is horizontal and the circular cap lies above it. We have to show that each isosceles triangle $x y z$, with $x y=x z$, is of the form $p_{i}, p_{j}, p_{k}$, where $1 \leq i<j<k \leq m$ and $p_{i} p_{j}=p_{j} p_{k}$; in other words, $x$ lies between $y$ and $z$. Assume otherwise, say $y$ and $z$ follow $x$ in clockwise order (the case when $y$ and $z$ precede $x$ in clockwise order is similar).

Put $\alpha=\widehat{x y z}$. Denote by $g$ and $e$ the points of intersection of the line $x y$ with the circle. Denote by $d$ and $f$ the points of intersection of the line $y z$ with the circle. See Fig. 1. Clearly, $\alpha<90^{\circ}$, as one of the equal angles of an isosceles triangle. On the other hand, as an angle formed by two intersecting chords

$$
\alpha=\frac{\alpha_{1}+\alpha_{2}}{2},
$$

where $\alpha_{1}, \alpha_{2}$ are the measures of the circular clockwise arcs $d e$ and $f g$. Clearly, $\alpha_{1} \geq 0^{\circ}$, and by the convexity of $P, \alpha_{2} \geq 180^{\circ}$ (i.e., $p_{1}$ is on or below the line $g e$, and $p_{m}$ is on or below the line $d f$ ). This gives $\alpha \geq 90^{\circ}$, which is a contradiction.

We say that $p$ determines an isosceles triangle $\Delta p q r$ if $p q=p r$; thus, an equilateral triangle is determined by all its three vertices. We also say that $\Delta p q r$ is determined by $P$ if $p, q, r \in P$.

Corollary 1. Let $P$ be a set of $m$ points in convex position inside a closed cap of a disk $C$ determined by a chord $p q$, such that $p, q \in P$. Then the number I of isosceles triangles determined by $P$ is at most $(m-1)^{2} / 4$. 
Proof. Let $j \leq(m+1) / 2$. We claim that $p_{j}$ determines at most $j-1$ isosceles triangles. Indeed, by Lemma 2 , there are no isosceles triangles $\Delta p_{j} p_{k_{1}} p_{k_{2}}$ determined by $p_{j}$, where $j<k_{1}, k_{2}$. Similarly, there are no isosceles triangles $\Delta p_{i_{1}} p_{i_{2}} p_{j}$ determined by $p_{j}$, where $i_{1}, i_{2}<j$. Assume that $\Delta p_{i} p_{j} p_{k_{1}}$ and $\Delta p_{i} p_{j} p_{k_{2}}$ are two isosceles triangles determined by $p_{j}$, where $i<j<k_{1}$ and $i<j<k_{2}$. Then $\Delta p_{j} p_{k_{1}} p_{k_{2}}$, where $j<k_{1}, k_{2}$, is also an isosceles triangles determined by $p_{j}$, contradicting the above observation. This proves our claim.

Similarly, if $j \geq(m+1) / 2, p_{j}$ determines at most $m-j$ isosceles triangles. Therefore, the total number of isosceles triangles determined by $P$ is bounded as follows:

For even $m$,

$$
I \leq 2 \sum_{2 \leq j \leq m / 2}(j-1)=\frac{(m-2) m}{4} \leq \frac{(m-1)^{2}}{4} .
$$

For odd $m$,

$$
I \leq 2 \sum_{2 \leq j \leq(m-1) / 2}(j-1)+\frac{m-1}{2}=\frac{(m-1)^{2}}{4}
$$

We continue with the proof of Theorem 2, and denote by $P$ a set of $n$ points in convex position. By Corollary 1 , for each $i \in\{1,2,3\}$, at least $\left(\begin{array}{c}m_{i} \\ 2\end{array}\right)-\left(m_{i}-1\right)^{2} / 4$ segments whose endpoints are in $C_{i}$ are the base of at most one isosceles triangle determined by $P$. Consequently, the number $I$ of isosceles triangles determined by $P$ is

$$
I \leq 2\left(\begin{array}{l}
n \\
2
\end{array}\right)-\sum_{i=1}^{3}\left\{\left(\begin{array}{c}
m_{i} \\
2
\end{array}\right)-\frac{\left(m_{i}-1\right)^{2}}{4}\right\} \leq 2\left(\begin{array}{l}
n \\
2
\end{array}\right)-\sum_{i=1}^{3} \frac{m_{i}^{2}-1}{4} .
$$

By the Cauchy-Schwarz inequality,

$$
\sum_{i=1}^{3} \frac{m_{i}^{2}-1}{4} \geq \frac{1}{4}\left(3\left(\frac{n+3}{3}\right)^{2}-3\right)=\frac{n^{2}+6 n}{12} .
$$

Plugging this bound into (4), we get that

$$
I \leq 2\left(\begin{array}{l}
n \\
2
\end{array}\right)-\frac{n^{2}+6 n}{12}=\frac{11 n^{2}-18 n}{12} .
$$

Comparing (2) and (5), one gets $k \gtrsim 6 n / 17$. We now obtain a better lower bound than (2), which in turn gives a better result. For a given $p \in P$, denote by $I(p)$ the number of isosceles triangles determined by $p$. We recall that $I(p)$ attains its minimum when the points of $P \backslash\{p\}$ are distributed on the (at most $k$ concentric) circles $C_{1}(p), \ldots, C_{k}(p)$ centered at $p$ as evenly as possible. Since we can assume that $\lceil n / 3\rceil \leq k \leq\lfloor n / 2\rfloor$, this means that each circle contains either two or three points. Let $a$ (resp. $b$ ) be the number of circles centered at $p$ that pass through exactly two (resp. three) points of $P \backslash\{p\}$. We have $a+b \leq k$ and $2 a+3 b=n-1$. Solving for $a$ and $b$ yields

$$
\begin{aligned}
& a \leq 3 k-(n-1), \\
& b \geq(n-1)-2 k .
\end{aligned}
$$


Then the number of isosceles triangles $I(p)$ determined by $p$ is

$$
I(p) \geq a+3 b=(2 a+3 b)-a \geq(n-1)-(3 k-(n-1))=2(n-1)-3 k,
$$

and, consequently,

$$
I=\sum_{p \in P} I(p) \geq n(2 n-2-3 k) .
$$

Comparing (5) and (6) yields

$$
k \geq\left\lceil\frac{13 n-6}{36}\right\rceil
$$

as promised.

\section{Concluding Remarks}

Let $t(p)$ be the number of distinct distances from vertex $p$ to the other vertices of a convex $n$-gon $P$. The $t$-sequence of $P$, viewed cyclically, is $\left(t\left(p_{1}\right), t\left(p_{2}\right), \ldots, t\left(p_{n}\right)\right)$ with $p_{1}, p_{2}, \ldots, p_{n}$ in clockwise (or counterclockwise) order. Let $T(P)=t\left(p_{1}\right)+$ $t\left(p_{2}\right)+\cdots+t\left(p_{n}\right)$, and

$$
T_{n}=\min \{T(P): P \text { is a convex } n \text {-gon }\} .
$$

We conclude by recalling two related conjectures on convex $n$-gons, C 2 and C 3 , posed by Erdős and Fishburn [6]-[8], vis-à-vis conjecture C1, the one discussed in this paper:

C1. (Erdős) Some vertex has at least $\lfloor n / 2\rfloor$ distinct distances to other vertices.

C2. (Fishburn) Each of at least $\lfloor n / 2\rfloor$ vertices has at least $\lfloor n / 2\rfloor$ distinct distances to other distances.

C3. (Erdős and Fishburn) $T_{n}=\left(\begin{array}{l}n \\ 2\end{array}\right)$ for all $n \geq 3$.

Clearly $\mathrm{C} 2$ is a strengthening of $\mathrm{C} 1$. Since $\mathrm{C} 3$ implies that the minimum average $t(p)$ is $(n-1) / 2, \mathrm{C} 3$ is a strengthening of $\mathrm{C} 1$ as well.

\section{References}

1. E. Altman, On a problem of Erdôs, American Mathematical Monthly, 70 (1963), 148-157.

2. E. Altman, Some theorems on convex polygons, Canadian Mathematical Bulletin, 15 (1972), 329-340.

3. P. Erdős, On sets of distances of $n$ points, American Mathematical Monthly, 53 (1946), 248-250.

4. P. Erdős, Some combinatorial and metric problems in geometry, in: Intuitive Geometry, Colloquia Mathematica Societatis János Bolyai, vol. 48 (K. Böröczky and G. Fejes Tóth, editors), North-Holland, Amsterdam, 1987.

5. P. Erdős and P. Fishburn, A postscript on distances in convex $n$-gons, Discrete \& Computational Geometry, 11 (1994), 111-117.

6. P. Erdős and P. Fishburn, Multiplicities of interpoint distances in finite planar sets, Discrete Applied Mathematics, 60 (1995), 141-147.

7. P. Erdôs and P. Fishburn, Intervertex distances in convex polygons, Discrete Applied Mathematics, 60 (1995), 149-158. 
8. P. Fishburn, Distances in convex polygons, in: The Mathematics of Paul Erdös, vol. 2 (R. Graham and J. Nešetřil, editors), Springer-Verlag, New York, 1996, pages 284-293.

9. N. Katz and G. Tardos, A new entropy inequality for the Erdős distance problem, in Towards a Theory of Geometric Graphs, János Pach (editor), Contemporary Mathematics, AMS, Providence, RI, 2004, pages 119-126.

10. L. Moser, On different distances determined by $n$ points, American Mathematical Monthly, 59 (1952), 85-91.

11. J. Pach and P. K. Agarwal, Combinatorial Geometry, Wiley, New York, 1995.

12. J. Pach and G. Tardos, Isosceles triangles determined by a planar point set, Graphs and Combinatorics, 18 (2002), 769-779.

13. J. Solymosi and C. Tóth, Distinct distances in the plane, Discrete \& Computational Geometry, 25 (2001), 629-634.

14. G. Tardos, On distinct sums and distinct distances, Advances in Mathematics, 180(1) (2003), $275-289$.

Received June 30, 2005. Online publication September 29, 2006. 